\title{
Perception of floral volatiles involved in host-plant finding behaviour: comparison of a bee specialist and generalist
}

\author{
Hannah Burger - Manfred Ayasse • \\ Stefan Dötterl · Sabine Kreissl • C. Giovanni Galizia
}

\begin{abstract}
Specialist and generalist bees use olfactory and visual cues to find and recognise flowering plants. Specialised (oligolectic) bees rely on few host plants for pollen collection. These bee species are suggested to use specific volatiles, but it is unknown whether they have dedicated adaptations for these particular compounds compared to bees not specialised on the same plants. In the present study, we investigated the perception of host odorants and its neuronal substrate with regard to host-plant finding behaviour in oligolectic bees. We reconstructed the antennal lobes (AL) in the Salix specialist, Andrena vaga, and counted about 135 glomeruli and thereby less than the approximately 160 in honeybees. Using calcium imaging experiments to measure neural activity in the bee brain, we recorded odorant-evoked activity patterns in the AL of A. vaga and, for comparison, in the generalist honeybee,
\end{abstract}

Electronic supplementary material The online version of this article (doi:10.1007/s00359 0130835 5) contains supplementary material, which is available to authorized users.

H. Burger $(\bowtie) \cdot$ M. Ayasse

Institute of Experimental Ecology, University of Ulm,

Albert Einstein Allee 11, 89069 Ulm, Germany

e mail: hannah.burger@uni ulm.de

\section{S. Dötterl}

Department of Plant Systematics, University of Bayreuth, Universitätsstraße 30, 95440 Bayreuth, Germany

\section{Present Address:}

S. Dötterl

Organismic Biology, Plant Ecology, Salzburg University,

Hellbrunnerstr. 34, 5020 Salzburg, Austria

\section{S. Kreissl · C. G. Galizia}

Department of Neurobiology, University of Konstanz,

78457 Konstanz, Germany
Apis mellifera. Our physiological experiments demonstrated that $A$. vaga bees were particularly sensitive to 1,4dimethoxybenzene, a behaviour-mediating odorant of Salix host flowers. We found more sensitive glomeruli in the specialised bees as compared to generalist honeybees. This neural adaptation might allow oligolectic A. vaga bees to effectively locate host plants from distances.

Keywords Olfaction - Specialised (oligolectic) bee · Host-plant odours - Calcium imaging - Antennal lobe morphology

\section{Introduction}

Floral volatiles are important mediators in the interaction between flowering plants and their pollinators. Floral scent plays a role in long-range orientation to flowers and can trigger landing and feeding behaviour of flower visitors (Raguso 2008b). Furthermore, pollinating insects use flower odours to recognise different plant species and to discriminate between rewarding and non-rewarding flowers within species (Dobson and Bergström 2000; Raguso 2008a).

Oligolectic bees collect pollen from only few related plant species. Even when such bees are reared on non-host pollen, they restrict pollen collection to their host, as demonstrated in Heriades truncorum (Megachilidae) (Praz et al. 2008). Furthermore, it is hypothesised that oligolectic bees are evolutionarily constrained: physiological adaptations to digest the pollen diet and/or neurological adaptations to recognise floral features may restrict specialised bees to their hosts (Sedivy et al. 2008).

Specialist bees are suggested to rely on taxon-specific olfactory cues to find their specific host plants (Dötterl and 
Vereecken 2010, and references therein). For example, oligolectic Hoplitis adunca (Megachilidae) females recognise their Echium host flowers by the highly specific floral volatile 1,4-benzoquinone, which is not known from other plants (Burger et al. 2010a, b, 2012). Newly emerged oligolectic bees (foraging-naïve) generally need to find host plants during their first foraging trips to reproduce successfully. While naïve bees initially respond to floral traits, experienced ones may rely additionally on learned features (Dobson and Bergström 2000; Dötterl and Vereecken 2010; Dötterl et al. 2011; Milet-Pinheiro et al. 2012).

Individuals of the pollen specialist, Andrena vaga (Andrenidae) (Fig. 1), were attracted by 1,4-dimethoxybenzene (Dötterl et al. 2005), a main compound of some Salix (willows) host species (e.g. S. caprea, S. cinerea, S. fragilis) (Tollsten and Knudsen 1992; Füssel et al. 2007). Additionally, minor compounds of the host odorants such as methyl salicylate, 2-phenylethanol and $(E, E)$ - $\alpha$-farnesene but not eugenol were also attractive (Dötterl and Vereecken 2010). As 1,4-dimethoxybenzene and the other compounds are not restricted to the floral scent of Salix host plants, these compounds are probably not the only signal used by $A$. vaga to recognise its host plants; however, they can play a key role in the host-finding process. Salix trees with thousands of flowers arranged in catkins emit a high amount of scent well suited as a long-distance attractant for bees collecting floral rewards on such trees. Salix flowers are an important food source not only for oligolectic bees but also for many other insect visitors including the polylectic honeybee, Apis mellifera (Füssel 2007). As the attractive floral volatiles are known attractants for A. mellifera as well (Füssel 2007; Dötterl and Vereecken 2010), this generalist bee might use the same floral compounds as A. vaga to find Salix trees.

In all hymenopteran species, odorants are detected by olfactory receptors on the antennae. All axons of olfactory receptor cells enter the brain and coalesce in the antennal

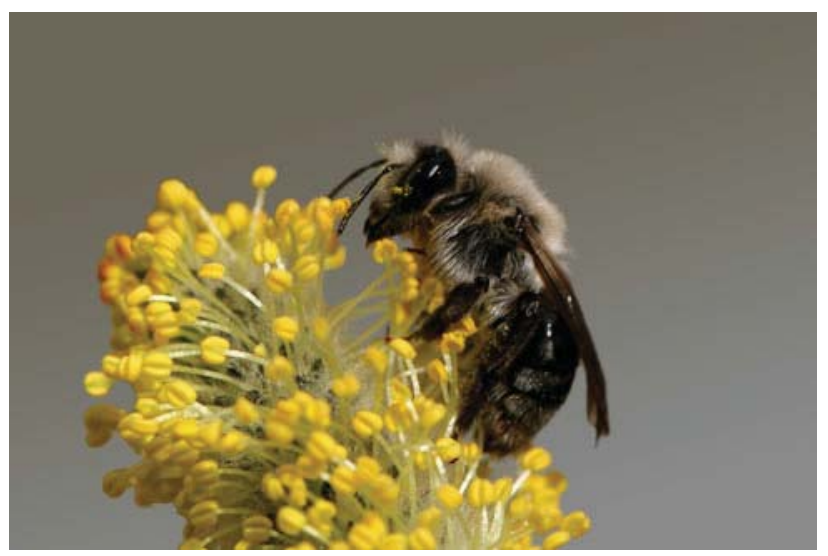

Fig. 1 Andrena vaga female visiting a catkin of Salix cinerea lobe (AL), the first brain structure to process olfactory information. Here, each family of receptor cells innervates a specific glomerulus, and therefore response patterns across receptor cell families are equivalent to activity patterns across olfactory glomeruli in the AL.

In this study, we tested whether the specialist biology of A. vaga is reflected in the morphology and physiology of the AL. Specifically, we hypothesise that a specialist might need fewer glomeruli, given a more restricted odour landscape, as compared to a generalist. Furthermore, we hypothesise that oligolectic $A$. vaga females might have evolved neural adaptations to localise host plants successfully. In particular, it should be advantageous for specialist bees to be highly sensitive for key compounds of their host plants. We performed calcium imaging experiments in ALs of specialist A. vaga and generalist honeybees to test whether oligolectic bee brains display different glomerular tuning compared to A. mellifera. We measured responses to two of the host odorants, 1,4-dimethoxybenzene and 2-phenylethanol. 1,4-Dimethoxybenzene was chosen for comparison as it is a typical component of some willows visited by both bee species (Tollsten and Knudsen 1992; Füssel et al. 2007) and 2-phenylethanol is one of the most widespread floral volatiles (Knudsen et al. 2006). Calcium imaging allows quantifying changes in intracellular calcium concentrations as a measure of odorant-evoked neuronal activity (Galizia and Menzel 2001). Amongst bees, this technique has been used to study odour coding in honeybees (Galizia et al. 1999b; Sachse and Galizia 2002) but in no other bee species so far.

A. vaga bees with a different status of foraging-experience responded differently to host-plant odorants in behavioural experiments (Dötterl et al. 2005; Dötterl and Vereecken 2010). As an explanation it was suggested that the experienced bees might be less attracted by odorant stimuli and instead use other cues (e.g. landmarks) to return to specific host-plant patches (Dötterl and Vereecken 2010). As changes in behaviour can be associated also to changes in the olfactory system (Anton et al. 2007), we recorded neuronal responses in naïve and in experienced bees and tested for differences between them. We complemented the physiological recordings with anatomical staining of olfactory sensory neurons (OSNs). We reconstructed the glomerular organisation in A. vaga and counted olfactory glomeruli for a comparison to A. mellifera.

\section{Materials and methods}

Study organisms

A. vaga is a ground-nesting solitary bee which is active in March May when Salix host plants are flowering. The 
geographic range extends from middle and north Europe to Central Asia (Westrich 1989). Bees were collected in the Botanical Garden of the University of Ulm, Germany, where a bee population had been established in a sandy nesting ground several years earlier. Before bees started to become active, males and females were carefully dug out and placed in boxes containing sand and stored in dark at $6{ }^{\circ} \mathrm{C}$ in a climate chamber. These bees were foragingnaïve. During the flying season of $A$. vaga further individuals with flower experience (pollen-carrying females) were caught. Around 20 bees each were kept in two individual flight cages $(60 \times 60 \times 60 \mathrm{~cm})$ with a $10 \mathrm{~cm}$ layer of (wet) sand on the ground for 310 days until they were used for optical imaging experiments. The flight cages were placed close to a window in the laboratory at room temperature. Sponges saturated with sugar water (Apiinvert, Südzucker AG, Mannheim/Ochsenfurt, Germany) were offered to the bees in the flight cage. Only female bees were used.

In contrast to oligolectic A. vaga, A. mellifera is a polylectic bee species visiting a wide variety of flowering plants. Salix is an important food source for honeybees in early spring when only few other plant species are flowering. Honeybees foraging on sugar water were collected from hives at the University of Konstanz. Bees were taken from outside hives when weather conditions allowed foraging by the bees or from hives kept indoors with controlled temperature $\left(16{ }^{\circ} \mathrm{C}\right.$ at night and $25^{\circ} \mathrm{C}$ during the day), light/dark cycle (12/12 h), and humidity (ca. $75 \%$ ). For insect preparation, individuals of both bee species were cooled on ice for several minutes until they stopped moving.

\section{Optical imaging experiments}

Neuronal responses in the ALs of $A$. vaga females (naïve $N=14$, experienced $N=8$ ) and A. mellifera workers $(N=8)$ to synthetic odorants of Salix plants were analysed using calcium imaging experiments (Galizia and Vetter 2005; Najar-Rodriguez et al. 2010) in the years 2008 and 2009 (February May).

\section{Insect preparation and staining}

Individual bees were mounted on a custom-made Plexiglas stage, fixed with wax and dissected using the technique described by Galizia and Vetter (2005). Briefly, after exposing the ALs, they were stained for $60 \mathrm{~min}$ by bathapplying $10 \mu \mathrm{l}$ of the dye Calcium Green $5 \mathrm{~N}$ AM (Molecular Probes, Invitrogen, Carlsbad, CA, USA) dissolved in saline with Pluronic and dimethylsulfoxide (DMSO) to the open brain cavity. This procedure leads to signals that combine the activities of several different types of neurons and possibly also glial cells. In honeybees, it has been shown that the prevailing signals represent primarily sensory neuron responses (Galizia and Vetter 2005). After staining, the excess dye was removed and the brain was washed in saline (in mM: $130 \mathrm{NaCl}, 6 \mathrm{KCl}, 4 \mathrm{MgCl}_{2}, 5$ $\mathrm{CaCl}_{2}, 160$ sucrose, 25 D-glucose, 10 Hepes free acid, $\mathrm{pH}$ 6.7, $500 \mathrm{mOsmol}$ ). The mouthparts of $A$. vaga were carefully removed to prevent movement artefacts and the brain was covered with a thin layer of transparent two-component silicon (KwikSil, World Precision Instruments, Sarasota, FL, USA). Within $1030 \mathrm{~min}$, the bees were placed under an upright microscope (Olympus BX50WI, Hamburg, Germany) with a $20 \times$ water-immersion physiology objective $(\mathrm{NA}=0.95$, Olympus XLUM Plan FI). The preparation was kept in a stream of temperature-controlled and charcoal-cleaned air (air speed: $1.3 \mathrm{~m} / \mathrm{s}, 2123{ }^{\circ} \mathrm{C}$ ) to avoid contamination from external odorants in the room.

\section{Odorant stimulation}

Compounds used to stimulate neural responses in AL for optical imaging experiments were 1,4-dimethoxybenzene, methyl salicylate, eugenol, 2-phenylethanol (all SigmaAldrich, $>99 \%$ ), and (E,E)- $\alpha$-farnesene (Sigma Aldrich, $>75 \%$ ). Four different dilutions at $10^{-2}, 10^{-3}, 10^{-4}$ and $10^{-5} \mathrm{v} / \mathrm{v}$ were prepared with each single odorant. Mineral oil (Fluka, $>99 \%$ ) was used as the solvent. In A. vaga, linalool (Sigma-Aldrich, $>97 \%$ ) at a concentration of $10^{-4}$ and in A. mellifera nonanol at a concentration of $10^{-2}$ was included as a reference odorant allowing normalisation of the magnitude of the calcium responses across tested females. Both reference odorants are widely distributed floral volatiles (Knudsen et al. 2006) that can be well perceived by the bees. The use of different reference odorants should not affect the results as statistical comparisons of normalised odorant responses were only done within a species. We did not directly compare the responses of A. vaga and A. mellifera in a statistical analysis. However, in a control analysis we recorded the response to linalool in four A. mellifera workers and normalised them using linalool (see online resource 1): this subset confirmed the results shown for the complete set with nonanol as reference odorant.

$5 \mathrm{ml}$ of the diluted odorants were kept in a $20 \mathrm{ml}$ glass vial filled with gaseous nitrogen to avoid chemical oxidation and sealed with aluminium ring caps fitted with a silicon/teflon septum (Axel Semrau, Sprockhövel, Germany) as described and detailed elsewhere (Pelz et al. 2006). As a control, a vial containing the solvent mineral oil only was used.

Odorant stimuli ( $2 \mathrm{ml}$ of odorant-loaded headspace from the $20 \mathrm{ml}$ vials) were applied at $1 \mathrm{ml} / \mathrm{s}$ using a computercontrolled autosampler (Combi PAL, CTC Analytics AG, 
Zwingen, Switzerland). Each stimulus was presented as double pulse of $1 \mathrm{~s}$ duration each with a $2 \mathrm{~s}$ gap, but reached the insect antenna with a delay of $1 \mathrm{~s}$ due to the distance the odorant travels between the injection site of the autosampler and the insect antenna. Odorants were tested as serial dilutions (odorant blocks), always starting with the lowest concentration of each odorant. The order of the odorants was randomly changed every time a new bee was tested in order to minimise sequence effects. The reference odorant and the solvent mineral oil served as controls and were presented after each odorant block.

\section{Optical recordings}

Images were acquired with a CCD camera (Imago QE, TILL Photonics, Gräfelfing, Germany) attached to the microscope. An $8 \times 8$ binning on chip was applied to a spatial sampling rate of $1.57 \times 1.57 \mu \mathrm{m} /$ pixel. Optical recordings consisted of 120 (A. vaga) or 80 (A. mellifera) frames taken for each stimulus at a frequency of $4 \mathrm{~Hz}$. The odorants were applied at 6 and $9 \mathrm{~s}$ after the recording has started which corresponded to frames 24 and 36. Excitation light was $470 \mathrm{~nm}$ and emission was filtered by a LP505 (Carl Zeiss GmbH, Hamburg, Germany). The exposure time was $20 \mathrm{~ms}$ per image (Polychrome V, intensity between 30 and $40 \%$, depending on the basal fluorescence values of the individual females).

\section{Signal calculation}

Imaging data were analysed using custom-written programmes in IDL (Research Systems, Inc., Boulder, CO, USA). Raw data were first corrected for lateral movement artefacts using anatomical landmarks. Then, data were logarithmically corrected for fluorescence intensity decay due to bleaching (Galizia and Vetter 2005) and filtered using a spatial median filter with a size of 5 pixels to reduce noise. An unsharp mask filter set to 2 pixels was applied to reduce scattered light produced by strongly activated glomeruli on neighbouring non-responding areas within the ALs (Galizia and Vetter 2005). The relative calcium change was then calculated for each frame as relative changes in fluorescence $(\Delta F / F)$. For the false colour images, the background fluorescence $(F)$ was defined as the fluorescence of frames 423 for every pixel and $\Delta F$ was defined as the difference between the fluorescence of frames 3436 and 6971 . Thus, each pixel was assigned a value that was then translated into a colour. Time traces for glomeruli were calculated from squares with a side length of 11 pixels and always well within each identified glomerulus.

For statistical comparison, each individual bee was normalised to the strongest linalool (A. vaga) or nonanol
(A. mellifera) response. Mann Whitney $U$ tests with Bonferroni Holm correction were performed to test for differences between odorant responses and responses to a solvent control. $P$ values were calculated by the Monte-Carlo method to avoid statistical failures because of different sample sizes in experiments with A. vaga (comparison of responses to odorants at a dilution of $10^{-5}$ : naïve $N=9$, experienced $N=4$, except 1,4-dimethoxybenzene naïve $N=14$, experienced $N=8$ individuals). The recordings were done from approximately 2025 glomeruli. Out of them one characteristic glomerulus was chosen for analysing the responses of each of the five odorants in each species. These glomeruli did not exclusively respond to one of the odorants but were regularly activated in most of the animals by the odorants at a dilution of $10^{-3}$. The responding glomeruli in $A$. mellifera were identified using the published atlas of honeybee AL (Galizia et al. 1999a). For visualisation of the differential odorant response patterns in A. vaga, responses to odorants at a low $\left(10^{-5}\right)$ and a medium concentration $\left(10^{-3}\right)$ were illustrated and analysed. To test for differences between the responses of naïve and experienced $A$. vaga females, a repeated-measures ANOVA for each odorant was carried out. The four different concentrations were treated as repeated measures and status (naïve/experienced) and glomerulus identity a e (naming see Fig. 2) were included as independent factors. The responses to 1,4-dimethoxybenzene and 2-phenylethanol at the four concentrations were chosen to compare responses between $A$. vaga and A. mellifera. The responses of naïve and experienced $A$. vaga individuals were pooled for comparison between species because the status of experience in Apis bees with regard to foraging on Salix was unknown.

\section{Morphology}

The right AL of three individual A. vaga females (naïve $N=2$, experienced $N=1$ ) were stained to reconstruct the morphology of glomeruli. The reconstruction enables the determination of the number and size of glomeruli. For comparison, we also stained the $\mathrm{AL}$ of one A. mellifera worker, and compared it to published material.

\section{Anatomical staining}

In order to visualise OSNs in all glomeruli, antennal afferents were stained as described elsewhere (Galizia et al. 1999a; Kreissl et al. 2010). Cooled bees were harnessed in plastic holders and the antennae were immobilised in a vertical position with eicosan. A well of Vaseline was built around the antennae and filled with $2 \%$ neurobiotin (Vector Laboratories, Burlingame, CA, USA) in distilled water. The antennae were cut at the base of the flagellum or at the pedicel and the well was sealed with Vaseline. Animals were kept in 
(a)

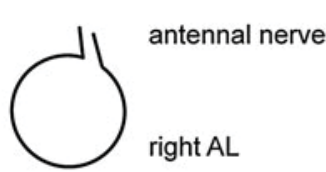

(b)

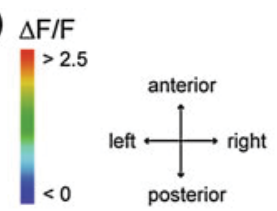

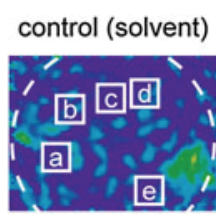

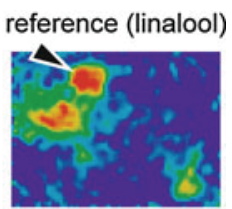

(c)

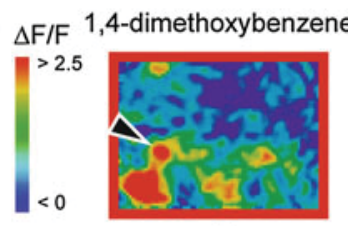

(d)

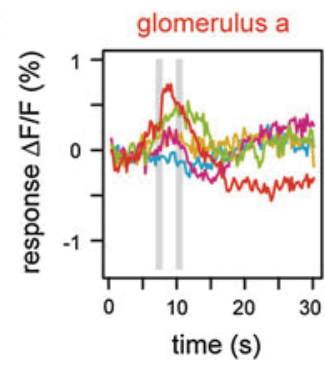

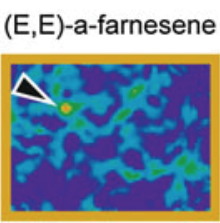

V

glomerulus b

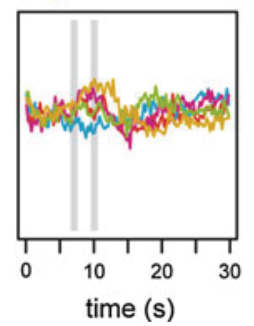

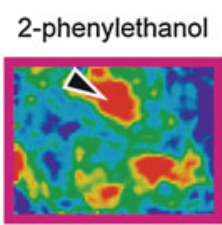

V

glomerulus c

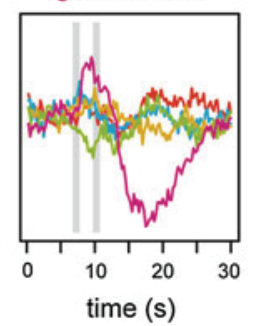

eugenol

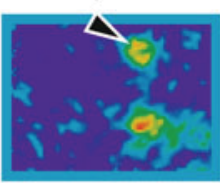

V

glomerulus d

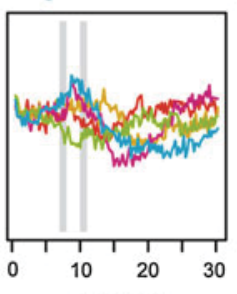

methyl salicylate

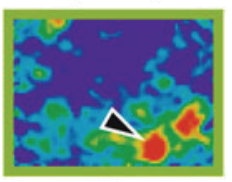

V

glomerulus e

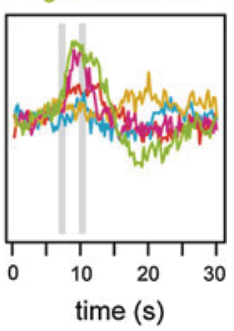

Fig. 2 Response patterns in the right antennal lobe (AL) of a representative A. vaga female using calcium imaging. a Schematic view of the AL ventral is up, medial (body axis) to the left. b False colour coded responses in control measurements (solvent control, linalool reference). The orientation and region of the antennal lobe and the selected glomeruli a e as well as the region of the glomerulus 1 (marked by arrowhead) used for normalisation are shown. c Glomerular response patterns for the five odorants 1,4 dimethoxy benzene, $(E, E) \propto$ farnesene, 2 phenylethanol, eugenol, and methyl

a moist dark chamber for $412 \mathrm{~h}$ at RT, after which the well containing the neurobiotin solution was carefully removed. Next, bee brains were dissected in $4 \%$ paraformaldehyde, transferred into fresh fixative and fixated for a total of $4 \mathrm{~h}$ at RT. The brains were washed at least five times in Triton X100 supplemented phosphate buffered saline (PBST $0.2 \%, 0.01 \mathrm{M}$ sodium phosphate buffer, $160 \mathrm{mM} \mathrm{NaCl}$, $0.2 \%$ Triton X100). To detect nuclei of cells in the AL and neurobiotin in the sensory fibres, DAPI (Sigma, St. Louis, MO, USA) and Cy3 streptavidin (Dianova, Hamburg) at a dilution of 1:500 in PBST 0.2\% Triton was applied. After thorough washing with PBST $0.2 \%$, brains were dehydrated in increasing concentrations of ethanol, cleared in xylene and mounted in DPX (Sigma-Aldrich).

\section{Confocal data analysis}

Whole mounts were scanned using a Zeiss LSM 510 NLO confocal microscope (Carl Zeiss, Jena, Germany). In confocal mode we used the laser line 543 and appropriate filter combinations. However, images slightly deteriorate with increasing depth. We therefore also used 2-photon excitation, a technique that allows deeper penetration into the tissue,

salicylate each in $10^{-3}$ dilution, showing that each odour elicits a different pattern (glomeruli a e are marked by arrowheads). d Time traces (normalised responses; colours of traces corresponds to colour labels of glomeruli a e) for each of the glomeruli a e, and the odorants shown in c. The times of odorant stimulations (double pulse of $1 \mathrm{~s}$ each) are indicated as grey bars in the time traces. Note the differences in response shape over time related to glomerulus odorant combination

while maintaining the spatial resolution of confocal microscopy. We used $750 \mathrm{~nm}$ to excite DAPI. Stacks were scanned using a $20 \times / 1.0$ water-immersion objective. Images were analysed and visualised using the Zeiss LSM image browser or AMIRA 5.2.1 (Visage Imaging, Berlin, Germany). Glomeruli of $A$. vaga were reconstructed using the segmentation editor in AMIRA. Single glomeruli were registered by interactive segmentation based on OSN mass fills. The volume of reconstructed glomeruli was used to estimate the mean diameter of glomeruli. As simplification, the reconstructed glomeruli were considered as balls, and the equation to calculate volume and radius of a sphere was used $\left(V=4 / 3 \pi r^{3}\right)$.

\section{Results}

\section{Odorant responses in Andrena vaga}

Each of the tested odorants induced calcium signals in the ALs of A. vaga females. These signals were spatially structured and corresponded to patterns consisting of individual glomeruli. 15 different glomeruli responded to the tested odorants. The spatial response patterns had a 
comparable topology across females. None of the responding glomeruli were found to be enlarged. In each pattern one characteristically responding glomerulus was chosen for further analysis (glomeruli a e). A representative individual with the selected glomeruli is illustrated in Fig. 2. The region of glomerulus 1 that responded across all individuals to the reference odorant linalool at a concentration of $10^{-4}$ is also exemplarily shown (Fig. 2b). A characteristic response in the other selected glomeruli was identified in at least $81.25 \%$ of the analysed animals.

1,4-Dimethoxybenzene evoked significantly higher and 2-phenylethanol significantly lower responses in naïve $A$. vaga females compared to experienced ones (responses of glomeruli to the four concentrations; Table 1; Fig. 3; see also online resource 2 for detailed statistical parameters). The responses to eugenol did not significantly differ between bees with different foraging experience status. $(E, E)-\alpha$-Farnesene and methyl salicylate evoked significantly different responses between naïve and experienced bees but the tendency for higher responses did vary for different concentrations and glomeruli. Compared to a solvent control only one glomerulus (glomerulus a) regularly and significantly responded to 1,4-dimethoxybenzene at $10^{-5}$ dilution in naïve individuals (Fig. 4a). All other glomeruli and odorants required higher odorant concentrations to yield responses significantly different from the solvent control (e.g. $10^{-3}$; Fig. 4).

Comparison of odorant responses between

Andrena vaga and Apis mellifera

No glomerulus in the ALs of A. mellifera was observed to respond to 1,4-dimethoxybenzene at the lowest tested concentration of $10^{-5}$. In contrast, glomerulus a in A. vaga had already a significant response to 1,4-dimethoxybenzene at $10^{-5}$ and $10^{-4}$ (Fig. 5a). Only one glomerulus (glomerulus A48) in A. mellifera responded significantly to 1,4-dimethoxybenzene at concentrations of $10^{-3}$ and $10^{-2}$
(Fig. 5b). A similar dose response pattern was observed for another glomerulus (glomerulus A28) characteristically responding to 2-phenylethanol in A. mellifera at the concentrations $10^{-3}$ and $10^{-2}$, but not at $10^{-5}$ and $10^{-4}$. Such a dose response relationship towards 2-phenylethanol was also found in the glomerulus $\mathrm{c}$ in the ALs of $A$. vaga (Fig. 5a). Glomerulus A17 in the ALs of A. mellifera responded consistently to the nonanol reference and was chosen for calculation of normalised data.

\section{AL morphology}

A morphological view of OSN axons in the AL of A. vaga and A. mellifera is shown in Fig. 6. The OSNs entered the AL via the antennal nerve from a anterior-lateral direction relative to the body axes in A. vaga and anterior-ventral in A. mellifera. In $A$. vaga, the nerve divided at the entrance in four main branches which subsequently split in numerous smaller subbranches. The sub-branches traversed the central neuropile of the AL and the sensory axons finally invaded the glomeruli (Figs. 6c, 7; online resource 3). Within a glomerulus, they mainly occupied a peripheral region but also ramified through the central part with a low density. Each of the four subbranches of the antennal nerve innervates one subgroup of glomeruli (Fig. 7; online resource 2). The reconstructed ALs of three analysed A. vaga females consisted of 135, 127 and 136 glomeruli with a mean glomeruli diameter of 39, 35 and $38 \mu \mathrm{m}$, respectively. We did not find any particularly enlarged glomerulus as it is the case in A. mellifera.

\section{Discussion}

Sensitivity of specialist A. vaga to host odorants

A high sensitivity to specific host volatiles may enable oligolectic $A$. vaga females to locate their host plants successfully. Using calcium imaging techniques, we found

Table 1 Statistics for comparison of odorant responses between naïve and experienced A. vaga females by means of repeated measures ANOVA $(* * P<0.001, * P<0.05$, ns $P>0.05)$

\begin{tabular}{|c|c|c|c|c|c|}
\hline & 1,4 Dimethoxybenzene & $(E, E) \propto$ Farnesene & 2 Phenylethanol & Eugenol & Methyl salicylate \\
\hline Intercept & $* *$ & $* *$ & $* *$ & $* *$ & $* *$ \\
\hline Glomerulus & $* *$ & $* *$ & $* *$ & $* *$ & $* *$ \\
\hline Status & $*$ & $*$ & $* *$ & ns & ns \\
\hline Glomerulus $\times$ status & ns & ns & ns & ns & ns \\
\hline Odorant concentration (repeated measures) & $* *$ & ** & $* *$ & $* *$ & $* *$ \\
\hline Odorant concentration $\times$ glomerulus & $* *$ & $* *$ & $* *$ & $* *$ & $* *$ \\
\hline Odorant concentration $\times$ status & $* *$ & ns & $* *$ & ns & $*$ \\
\hline Odorant concentration $\times$ glomerulus $\times$ status & ns & ns & ns & ns & ns \\
\hline
\end{tabular}

Glomerulus: effect among glomeruli a e, status: effect between naïve and experienced individuals, odorant concentration: effect among the odorant concentrations $10^{-5}, 10^{-4}, 10^{-3}$, and $10^{-2}$ 
(a) 1,4-dimethoxybenzene

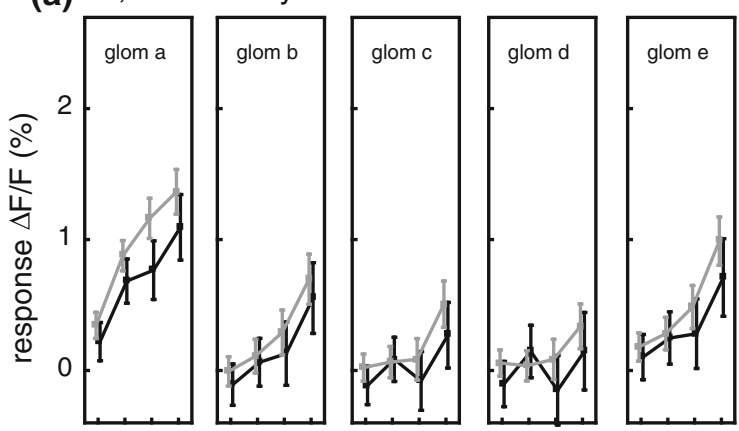

(c) 2-phenylethanol

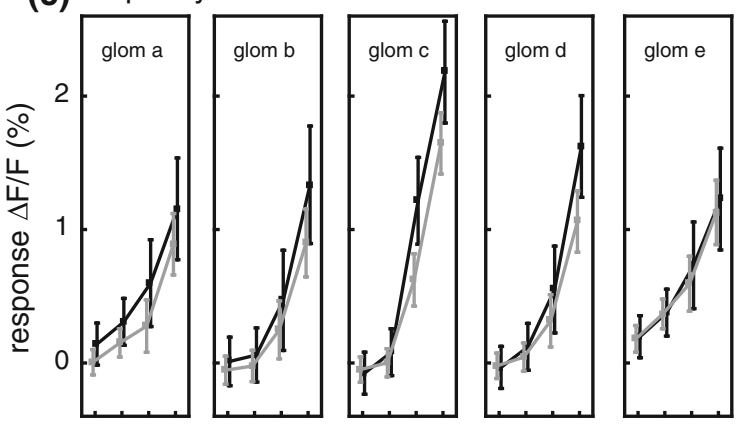

(e) methyl salicylate

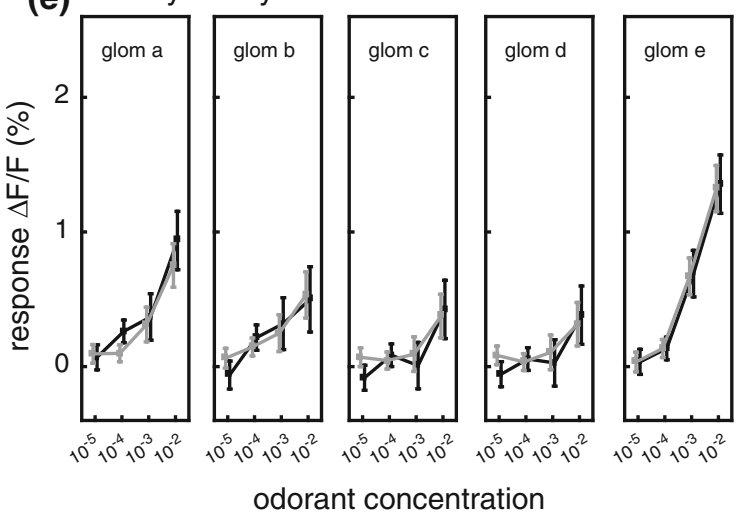

(b)

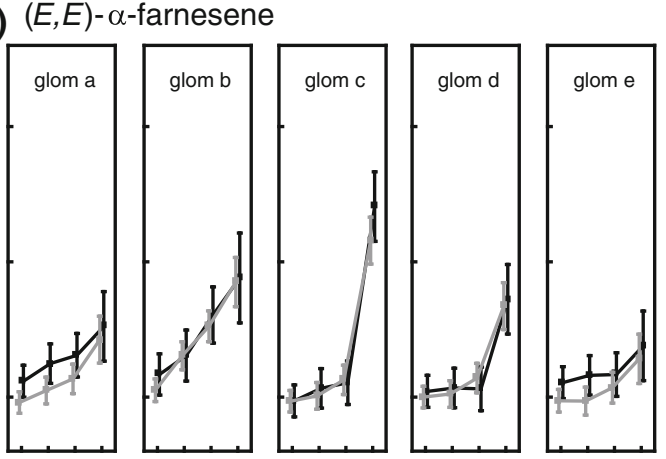

(d) eugenol

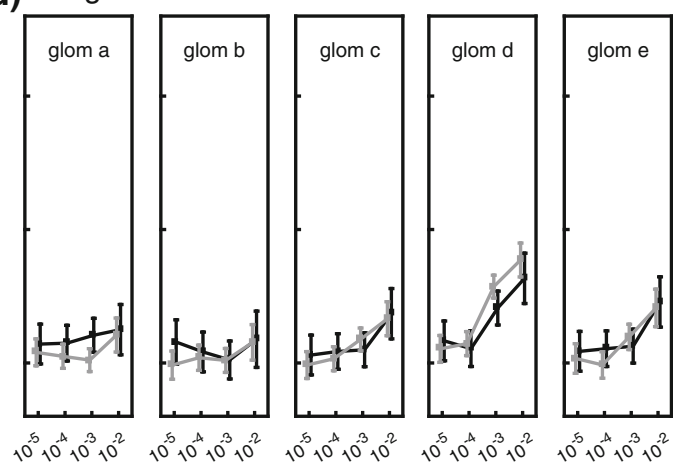

odorant concentration

naïve Andrena

- experienced Andrena
Fig. 3 Comparison of responses between naïve and experienced $A$. vaga females. Responses were recorded to a 1,4 dimethoxybenzene, b $(E, E) \alpha$ farnesene, c 2 phenylethanol, d eugenol, and e methyl salicylate each in $10^{-5}, 10^{-4}, 10^{-3}$, and $10^{-2}$ concentrations within

that bees show high sensitivities in the primary olfactory centre, the AL, for behaviour-mediating odorants identified in Salix (willows) host flowers. The high sensitivity to this aromatic compound might allow A. vaga to detect even trace amounts of this compound and help bees to locate host plants from large distances.

1,4-Dimethoxybenzene might be especially important for foraging-naïve bees as this main compound of Salix host flowers was the only compound in our experiments that elicited significant responses at the lowest measured concentration $\left(10^{-5}\right)$ recorded in naïve bees. Considering all the glomeruli (glom) a e (least square means, bars denote 0.95 confidence intervals; see Table 1 for statistical comparison using repeated measures ANOVA)

tested odorant concentrations, naïve bees were more sensitive for 1,4-dimethoxybenzene as compared to experienced ones. The recorded differences regarding the sensitivity to perceive host-flower volatiles between both bee groups stand in correlation to reported changes in behaviour of naïve and experienced A. vaga. While naïve bees were attracted by 1,4-dimethoxybenzene in behavioural experiments, bees with foraging-experience behaved differently and were no longer attracted (Dötterl et al. 2005). Foraging bees have already localised host-plant patches and probably orient on other cues like landmarks to return to pollen 
(a) naïve Andrena
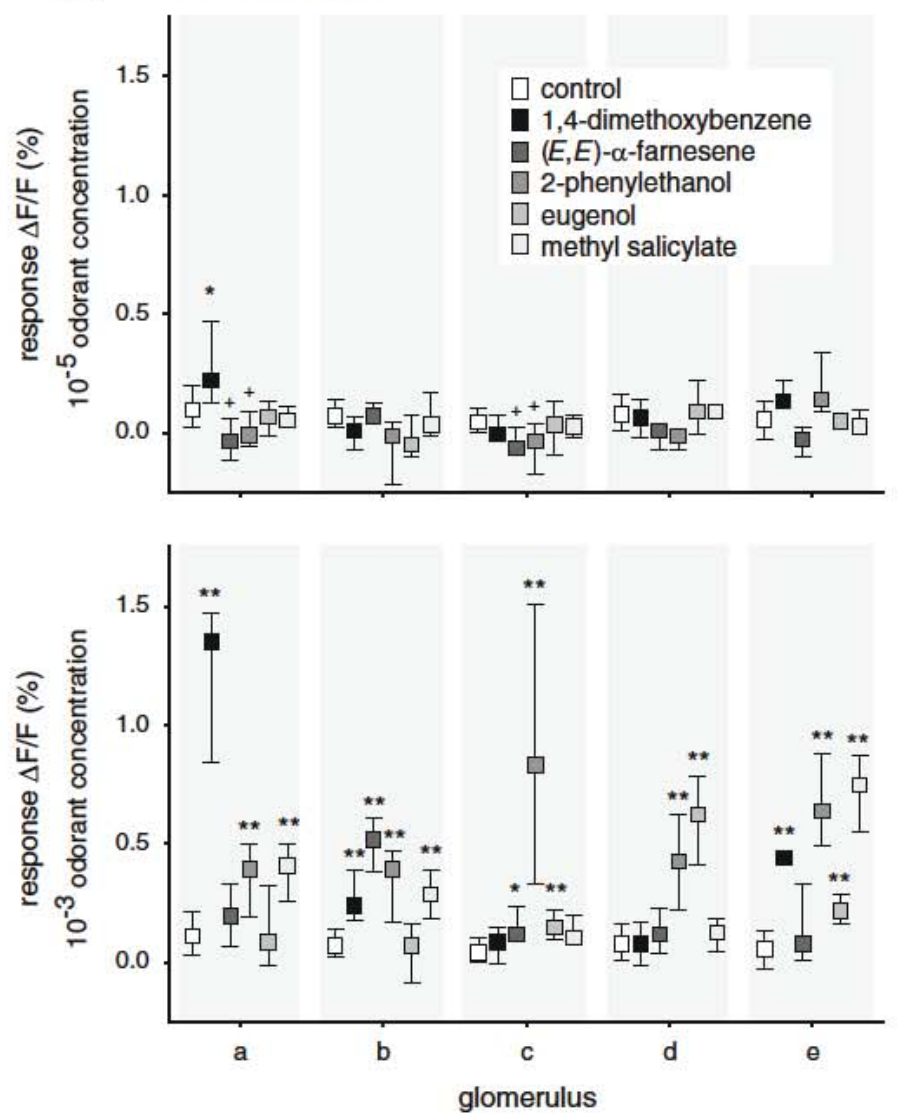

Fig. 4 Responses (median and whiskers $2575 \%$ of normalised data) of five selected glomeruli of a foraging naïve and bexperienced A. vaga to stimulations with air (control), 1,4 dimethoxybenzene, eugenol, $(E, E) \propto$ farnesene, 2 phenylethanol and methyl salicylate in $10^{-5}$ (naïve $N \quad 9$, experienced $N \quad 4$, except 1,4 dimethoxybenzene naïve

(a)

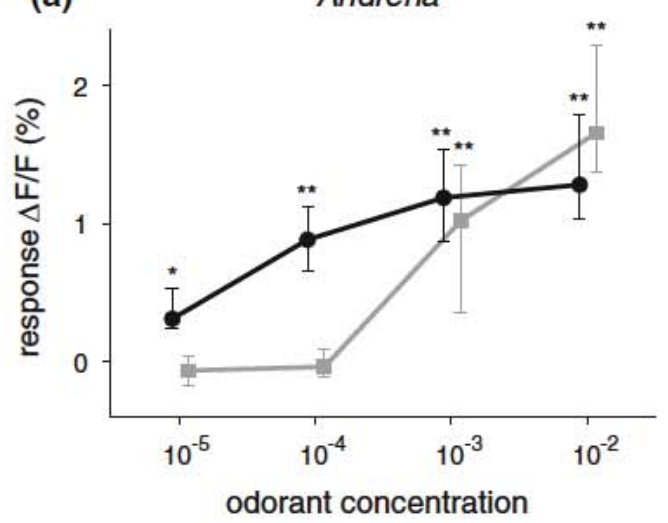

Fig. 5 Comparison between dose response relationships (median, whiskers $2575 \%$ of normalised data) in a A. vaga (1,4 dimethoxy benzene $N \quad 22,2$ phenylethanol $N \quad 13$ individuals) and b $A$. mellifera ( $N \quad 8$ individuals) bees responding to 1,4 dimethoxyben zene (glomerulus a in Andrena and A48 in Apis) and 2 phenylethanol (b) experienced Andrena
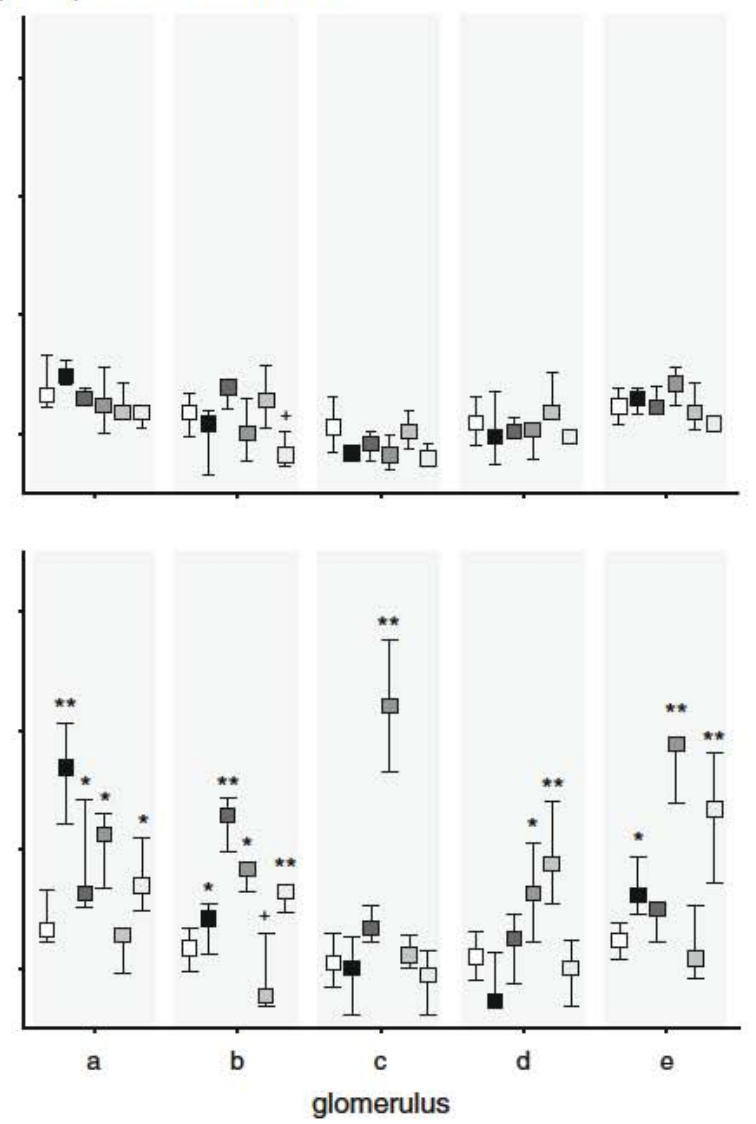

$N \quad 14$, experienced $N \quad 8$ individuals) and $10^{-3}$ (naïve $N \quad 14$, experienced $N \quad 8$ individuals) dilutions. Asterisks indicate a signif icantly higher, crosses a lower response compared to the control within each glomerulus ( $U$ test, $P$ calculated by Monte Carlo method, Bonferroni Holm correction, ${ }^{*} P<0.01,{ }^{* *} P<0.001$ )

(b) Apis

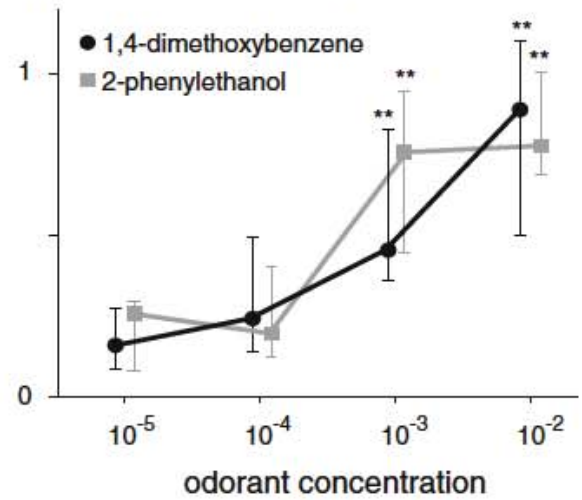

(glomerulus c in Andrena and A28 in Apis). Asterisks indicate a significantly higher response ( $U$ test, $P$ calculated by Monte Carlo method, Bonferroni Holm correction, $* P<0.01$, $* * P<0.001$ ) compared to stimulations with a solvent control (not shown) 

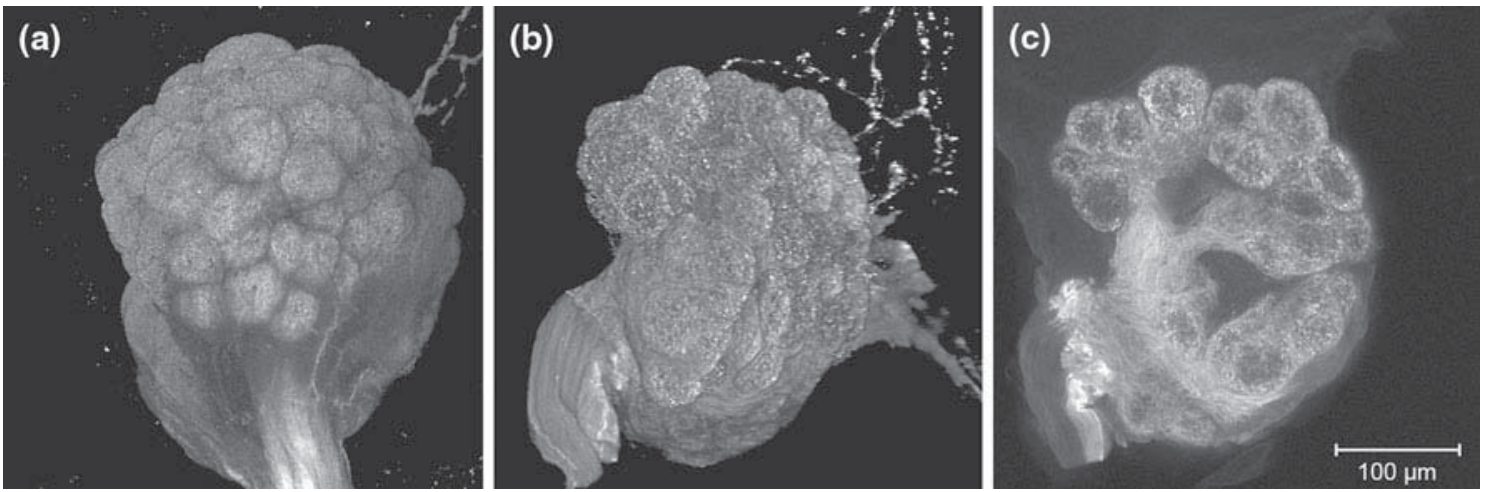

Fig. 6 Frontal views of the antennal lobes of an A. mellifera worker and $A$. vaga female. Lateral is to the left, medial to the right, dorsal is up, ventral down. Full maximal intensity projection of a complete stack of optical slices capturing the ALs in a A. mellifera and $\mathbf{b} A$. vaga. The antennal nerve enters the $\mathrm{AL}$ from anterior lateral in $A$. vaga and anterior ventral in A. mellifera. c Single optical slice $(10 \mu \mathrm{m}$ thick) at $60 \mu \mathrm{m}$ depth from the anterior surface of the AL showing some of the antennal nerve sub branches traversing the central neuropile of the AL of A. vaga

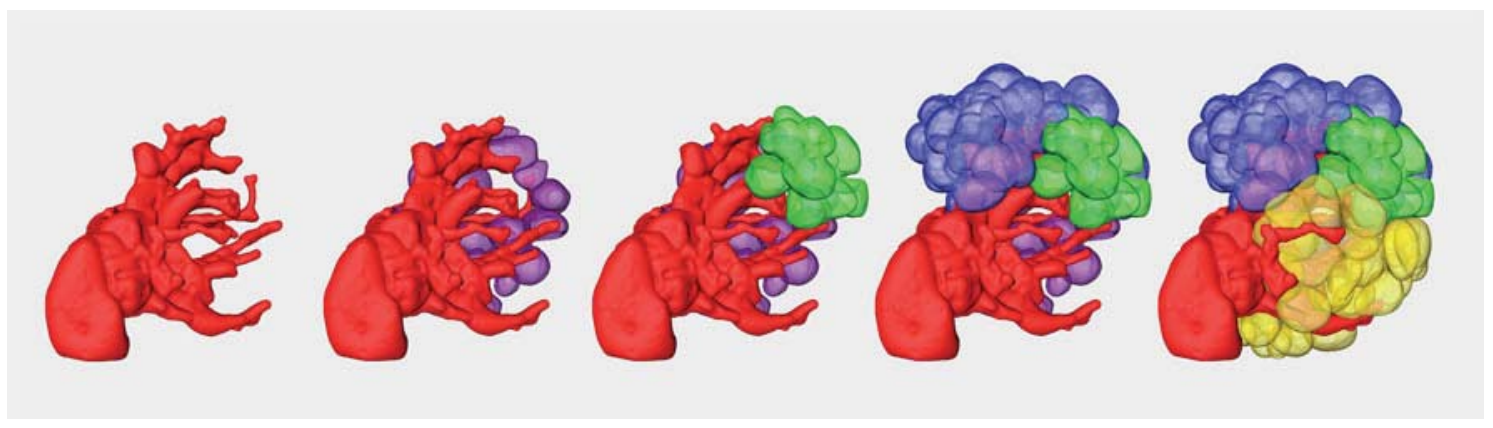

Fig. 7 Reconstruction of the right AL of an A. vaga female in an anterior view, ventral down, lateral left (compare with Fig. 6). The left image shows only the antennal nerve with its dividing branches within the central neuropile of the $\mathrm{AL}$ (red). In the following images the four groups of glomeruli innervated by the four main branches were added from posterior to anterior to create a three dimensional picture of glomerular positions (purple, green, blue, yellow) sources (Dötterl and Vereecken 2010). Therefore, floral volatiles for long-distance attraction might be less important for these experienced bees, in contrast to naive ones searching for host flowers on their very first foraging trips. In addition to learned landmarks, they might learn the more complex odour bouquet of the flowers, which is a more selective cue. Whether innate preference for a key substance is really replaced by a learned preference of an odour bouquet remains to be tested experimentally. This hypothesis is supported by our observation that experienced bees responded more to another floral volatile, 2-phenylethanol, in our imaging experiments as compared to naïve ones, even though they were not attracted by this compound in bioassays once they had gained foraging-experience (Dötterl and Vereecken 2010). As 2-phenylethanol is one of the most widespread floral volatiles (Knudsen et al. 2006) experienced $A$. vaga bees might use it in combination with further floral cues (e.g. a complete odour bouquet, or including other modalities such as floral colour) to find nectar sources. We conclude from these observations that further neural changes may occur in experienced A. vaga which can cause the reported altered behaviour. We assume that experience very likely affects further olfactory processing in brain neural networks, possibly including those in the AL. Indeed, plasticity in the honeybee AL has been shown even for nondevelopmental learning (Arenas et al. 2009; Fernandez et al. 2009; Rath et al. 2011). Still, measurements of the projection neurons (the output neurons in the AL), or measurements in higher brain centres such as the mushroom bodies, are needed to compare naïve and experienced animals to reveal the neural correlate of altered behaviour.

Comparison between specialist A. vaga and generalist A. mellifera

In comparison to the polylectic bee species A. mellifera, we found more sensitive glomeruli for the key compound 1,4dimethoxybenzene in A. vaga. Our approach, however, did 
not allow us to record responses from the opposite side of the ALs. Thus, we cannot exclude that A. mellifera may have one or more glomeruli that are as sensitive to 1,4dimethoxybenzene as the frontal glomerulus in A. vaga. While general properties of response patterns were equal across the two species investigated (i.e. odorants elicited patterned responses across glomeruli, and the time-courses of these calcium responses were equal), we did not find that the spatial pattern elicited by any of the odorants tested was equal across species, while, within a species, corresponding glomeruli were easy to identify. Two explanations are possible: either the specific response ranges of individual glomeruli have changed in evolution as exemplified by the differences in sensitivity that we found, or the spatial view onto the $\mathrm{AL}$ in our experiments was different due to morphological differences in the head-capsule arrangement, so that in fact the field of view differed. This is supported by the observed differences in AL morphology. Compared to A. mellifera, the antennal nerve invades the $\mathrm{AL}$ from a more lateral direction. It is thus likely that corresponding glomeruli in the two species are generally shifted along an oblique vertical axis. More odorantresponse measurements including many more glomeruli and possibly the creation of a 3D-atlas of olfactory glomeruli in $A$. vaga would be necessary, in order to compare this species in detail to A. mellifera (Galizia et al. 1999a).

Beside differences in the position of the AL, both species differ obviously in the number of glomeruli. Honeybees having about 160 glomeruli (Galizia et al. 1999a) exceed $A$. vaga with 135,127 and 136 counted glomeruli in three analysed individuals, respectively. The difference in glomerular number also indicates that it might be difficult to find homologous glomeruli solely based on their position in the AL. The reduced number of glomeruli in A. vaga might be correlated with the degree of floral specialisation: an oligolectic bee might need fewer floral odours to survive. However, although polylectic A. mellifera visits a broader range of flowers, this species shows also a considerably higher social structure. Foraging on flowers as well as social communication affords complex olfaction which might result in a higher number of glomeruli. The latter view is supported by the very high number of glomeruli in many ant species (Zube et al. 2008; Kelber et al. 2009). Further comparisons between solitary bee species are needed to reveal a correlation of glomeruli number with the range of host flowers.

Neural adaptations in correlation with host-finding behaviour

The specialised A. vaga showed a high sensitivity for 1,4dimethoxybenzene. This substance is a behaviour-releasing
(Dötterl et al. 2005) and characteristic floral volatile for some Salix host species (Tollsten and Knudsen 1992; Füssel et al. 2007). Our experiments, therefore, suggest that A. vaga females show correlates between neural organisation and host-plant finding behaviour. Among sensory modalities, adaptations to recognise host plants have rarely been studied in oligolectic bees. An exception is the colour vision of Callonychium petuniae specialised on purple coloured Petunia flowers and being most likely adapted to effectively detect these flowers using not only receptors most sensitive in the UV, blue, and green spectrum range, but additionally a red-biassed receptor (Cure and Wittmann 1990; Peitsch et al. 1992). In nymphalid butterflies with diverging host-plant ranges for oviposition, it was recently shown that odorant responses in ALs were mostly similar between two tested species but the specialist Aglais urticae was more sensitive for host-plant related odorants than the generalist Polygonia c-album (Carlsson et al. 2011).

In conclusion, we report a correlation between neural organisation and host-plant finding behaviour in the oligolectic bee A. vaga. A. vaga seems to have evolved neural adaptations to effectively locate host plants from distances being especially highly sensitive to the key component $1,4-$ dimethoxybenzene of the complex host-plant odour bouquet.

Acknowledgments We gratefully acknowledge B. Rapp who per formed the calcium imaging experiments on Apis mellifera, as well as M. Strauch, C. Lutz, and D. Münch who provided statistical tools, and M. Kaminski for the bee photograph. Two anonymous reviewers gave valuable comments on earlier versions of this manuscript. H. B. wishes to thank the Studienstiftung des deutschen Volkes for financial support. All experiments comply with the current laws of the country in which they were performed: in this case, Germany.

\section{References}

Anton S, Dufour M C, Gadenne C (2007) Plasticity of olfactory guided behaviour and its neurobiological basis: lessons from moths and locusts. Entomol Exp Appl 123:1 11

Arenas A, Giurfa M, Farina WM, Sandoz JC (2009) Early olfactory experience modifies neural activity in the antennal lobe of a social insect at the adult stage. Eur J Neurosci 30:1498 1508

Burger H, Ayasse M, Häberlein CM, Schulz S, Dötterl S (2010a) Echium and Pontechium specific floral cues for host plant recognition by the oligolectic bee Hoplitis adunca. S Afr J Bot 76:788 795

Burger H, Dötterl S, Ayasse M (2010b) Host plant finding and recognition by visual and olfactory floral cues in an oligolectic bee. Funct Ecol 24:1234 1240

Burger H, Dötterl S, Häberlein CM, Schulz S, Ayasse M (2012) An arthropod deterrent attracts specialised bees to their host plants. Oecologia 168:727 736

Carlsson MA, Bisch Knaden S, Schäpers A, Mozuraitis R, Hansson BS, Janz N (2011) Odour maps in the brain of butterflies with divergent host plant preferences. PLoS One 6:e24025 
Cure JR, Wittmann D (1990) Callonychium petuniae, a new panurgine bee species (Apoidea, Andrenidae) oligolectic on Petunia (Solanaceae). Stud Neotroph Fauna Environ 25:153 156

Dobson HEM, Bergström G (2000) The ecology and evolution of pollen odours. Plant Syst Evol 222:63 87

Dötterl S, Vereecken NJ (2010) The chemical ecology and evolution of bee flower interactions: a review and perspectives. Can J Zool 88:668 697

Dötterl S, Füssel U, Jürgens A, Aas G (2005) 1,4 Dimethoxybenzene, a floral scent compound in willows that attracts an oligolectic bee. J Chem Ecol 31:2993 2998

Dötterl S, Milchreit K, Schäffler I (2011) Behavioural plasticity and sex differences in host finding of a specialized bee species. J Comp Physiol A 197:1119 1126

Fernandez PC, Locatelli FF, Person Rennell N, Deleo G, Smith BH (2009) Associative conditioning tunes transient dynamics of early olfactory processing. J Neurosci 29:10191 10202

Füssel U (2007) Floral scent in Salix L. and the role of olfactory and visual cues for pollinator attraction of Salix caprea L. Disser tation, Universität Bayreuth

Füssel U, Dötterl S, Jürgens A, Aas G (2007) Inter and intraspecific variation in floral scent in the genus Salix and its implication for pollination. J Chem Ecol 33:749 765

Galizia CG, Menzel R (2001) The role of glomeruli in the neural representation of odours: results from optical recording studies. J Insect Physiol 47:115 130

Galizia CG, Vetter RS (2005) Optical methods for analyzing odour evoked activity in the insect brain. In: Christensen TA (ed) Methods in insect sensory neuroscience. CRC Press, Boca Raton, pp 349392

Galizia CG, McIlwrath SL, Menzel R (1999a) A digital three dimensional atlas of the honeybee antennal lobe based on optical sections acquired by confocal microscopy. Cell Tissue Res 295:383 394

Galizia CG, Sachse S, Rappert A, Menzel R (1999b) The glomerular code for odor representation is species specific in the honeybee Apis mellifera. Nat Neurosci 2:473 478

Kelber C, Rössler W, Roces F, Kleineidam C (2009) The antennal lobes of fungus growing ants (Attini): neuroanatomical traits and evolutionary trends. Brain Behav Evol 73:273 284

Knudsen JT, Eriksson R, Gershenzon J, Stahl B (2006) Diversity and distribution of floral scent. Bot Rev 72:1 120
Kreissl S, Strasser C, Galizia CG (2010) Allatostatin immunoreactiv ity in the honeybee brain. J Comp Neurol 518:1391 1417

Milet Pinheiro P, Ayasse M, Schlindwein C, Dobson H, Dötterl S (2012) Host location by visual and olfactory floral cues in an oligolectic bee: innate and learned behavior. Behav Ecol 23:531 538

Najar Rodriguez AJ, Galizia CG, Stierle J, Dorn S (2010) Behavioral and neurophysiological responses of an insect to changing ratios of constituents in host plant derived volatile mixtures. J Exp Biol 213:3388 3397

Peitsch D, Fietz A, Hertel H, de Souza J, Ventura DF, Menzel R (1992) The spectral input systems of Hymenopteran insects and their receptor based colour vision. J Comp Physiol A 170:23 40

Pelz D, Roeske T, Syed Z, de Bruyne M, Galizia CG (2006) The molecular receptive range of an olfactory receptor in vivo (Drosophila melanogaster Or22a). J Neurobiol 66:1544 1563

Praz CJ, Müller A, Dorn S (2008) Host recognition in a pollen specialist bee: evidence for a genetic basis. Apidologie 39:547 557

Raguso RA (2008a) Start making scents: the challenge of integrating chemistry into pollination ecology. Entomol Exp Appl 128:196 207

Raguso RA (2008b) Wake up and smell the roses: the ecology and evolution of floral scent. Ann Rev Ecol Evol Syst 39:549 569

Rath L, Galizia CG, Szyszka P (2011) Multiple memory traces after associative learning in the honey bee antennal lobe. Eur J Neurosci 34:352 360

Sachse S, Galizia CG (2002) Role of inhibition for temporal and spatial odor representation in olfactory output neurons: a calcium imaging study. J Neurophysiol 87:1106 1117

Sedivy C, Praz CJ, Müller A, Widmer A, Dorn S (2008) Patterns of host plant choice in bees of the genus Chelostoma: the constraint hypothesis of host range evolution in bees. Evolution 62:2487 2507

Tollsten L, Knudsen JT (1992) Floral scent in dioecious Salix (Salicaceae): a cue determining the pollination system? Plant Syst Evol 182:229 237

Westrich P (1989) Die Wildbienen Baden Württembergs. Eugen Ulmer, Stuttgart

Zube C, Kleineidam C, Kirschner S, Neef J, Rössler W (2008) Organization of the olfactory pathway and odor processing in the antennal lobe of the ant Camponotus floridanus. J Comp Neurol $506: 425441$ 